\title{
Self-assembled nanorings in Si-capped Ge quantum dots on (001)Si
}

\author{
S. W. Lee and L. J. Chen ${ }^{\text {a) }}$ \\ Department of Materials Science and Engineering, National Tsing Hua University, Hsinchu, \\ Taiwan, Republic of China \\ P. S. Chen, M.-J. Tsai, and C. W. Liu \\ Electronics Research and Service Organization, Industrial Technology Research Institute, Hsinchu, \\ Taiwan, Republic of China \\ T. Y. Chien and C. T. Chia \\ Department of Physics, National Taiwan Normal University, Taipei, Taiwan, Republic of China
}

(Received 19 March 2003; accepted 27 October 2003)

\begin{abstract}
Nanorings with an average height and diameter of 1.2 and $65 \mathrm{~nm}$, respectively, were observed to form in Si-capped Ge quantum dots grown at $600^{\circ} \mathrm{C}$ by ultrahigh-vacuum chemical vapor deposition. The nanorings were captured with the rapid cooling of the samples with appropriate amount of $\mathrm{Si}$ capping. Based on the results of transmission electron microscopy and Raman spectroscopy, the formation of nanorings is attributed to alloying and strain relief in the $\mathrm{Si} / \mathrm{Ge} /$ (001)Si system. The self-assembly of nanorings provides a useful scheme to form ultrasmall ring-like structure and facilitates the characterization of the physical properties of unconventional quantum structures. (C) 2003 American Institute of Physics. [DOI: 10.1063/1.1635073]
\end{abstract}

The growth of self-assembled Ge quantum dots (QDs) on $\mathrm{Si}$ has been intensively investigated for the promising applications in future electronic and optoelectronic devices in recent years. ${ }^{1-5}$ The structural and optical properties of QDs strongly depend on growth condition as well as the capping layer material and the growth process. ${ }^{6,7}$ However, the Ge QDs must be covered with a Si capping layer in order to be used in optoelectronic devices. ${ }^{8}$ Earlier studies indicate some structural changes of the Ge QDs during capping with $\mathrm{Si}^{9}{ }^{9,10}$ In a systematic investigation of the evolution of the Ge QDs during the initial stages of $\mathrm{Si}$ encapsulation up to 28 equivalent monolayers (eq-MLs), drastic changes of shape and surface morphology of the Ge QDs have been observed. In particular, nanorings were observed to form. The nanorings were captured with the rapid cooling of the samples with appropriate amount of Si capping. The mechanism for the self-assembly of an unconventional structure is suggested. Note that the amount of Ge deposition is expressed in the unit of equivalent monolayers (eq-MLs, 1 eq-ML $=6.27$ $\times 10^{14} \mathrm{Ge}$ atom $\left./ \mathrm{cm}^{2}\right) .{ }^{11}$

10-25 $\Omega \mathrm{cm}, 100 \mathrm{~mm}$ diameter $p$-type (001)-oriented $\mathrm{Si}$ wafers were used in the present study. All the Ge QDs investigated in this work were grown at $600{ }^{\circ} \mathrm{C}$ in a commercially available multiwafer ultrahigh-vacuum chemical vapor deposition (UHV/CVD) system. Pure $\mathrm{SiH}_{4}$ and $5 \% \mathrm{GeH}_{4}$ diluted in He were used as precursors. The Si wafers were dipped in a $10 \% \mathrm{HF}$ solution to achieve the $\mathrm{H}$ passivation, then transferred into the UHV/CVD system. A 60-nm-thick Si buffer layer was first grown. After depositing the Si buffer, a 13.1 eq-ML Ge layer was then deposited. To investigate the evolution of the Ge dots structure during the $\mathrm{Si}$ overgrowth, the coverages of Si capping layer on Ge islands were chosen to be $0,14,21,28$, and 56 eq-MLs. As the deposition was terminated, the wafers were kept in the deposition chamber

${ }^{a)}$ Electronic mail: ljchen@mse.nthu.edu.tw for 3 min, unless otherwise specified, to evacuate the residual reactant and were then removed from the chamber.

The Ge dot shape and size distribution were characterized by atomic force microscopy (AFM) in tapping mode. Both planview and cross-section transmission electron microscopy (XTEM) images revealed more detailed information about diameter and facets of the Ge nanostructures. TEM in conjunction with an energy dispersion spectrometer (EDS) was utilized to determine the composition of Ge nanostructures. For the EDS analysis the electron beam can be converged to a size as small as $1.5 \mathrm{~nm}$. Micro-Raman measurements were performed at room temperature. $\mathrm{Ar}^{+}$ion laser, $514.5 \mathrm{~nm}$ in wave length, with a power of $\sim 10 \mathrm{~mW}$ and a spot size of $\sim 1 \mu \mathrm{m}$ was used for excitation. High dispersion mode (or additive mode) was used to measure the $\mathrm{Si}-\mathrm{Ge}$ vibrations near $410 \mathrm{~cm}^{-1}$. The resolution of Raman spectra was approximately $0.1 \mathrm{~cm}^{-1}$.

The AFM images in Fig. 1 illustrate the evolution of the surface morphology during the overgrowth with $0-28$ eqMLs of Si. For an uncapped sample, Fig. 1(a) shows the well-known bimodal islands, pyramids with shallow $\{105\}$ facets and domes with higher angle facets, which are commonly observed for high temperature deposition. ${ }^{6,7}$ Roughly $88 \%$ of the Ge QDs are domes; the rest are pyramids. After deposition of 14 eq-MLs Si on Ge QDs, the pyramids are dominant and the domes are hardly seen, as shown in Fig. 1(b). In the mean time, the density of the QDs increased from about $9 \times 10^{9}-1.1 \times 10^{10} \mathrm{~cm}^{-2}$ with a broader islandsize distribution. The dome to pyramid shape transition of $\mathrm{Ge}$ QDs has been reported by Rastelli et al. ${ }^{9}$ This phenomenon was interpreted in terms of the dependence of equilibrium shape of QDs on their volume and composition as a result of intermixing.

After growth of 21 eq-ML Si as shown in Fig. 1(c), some small QDs disappear and the large QDs decrease further in height and diameter with a density of $7.9 \times 10^{9} \mathrm{~cm}^{-2}$. Figure 


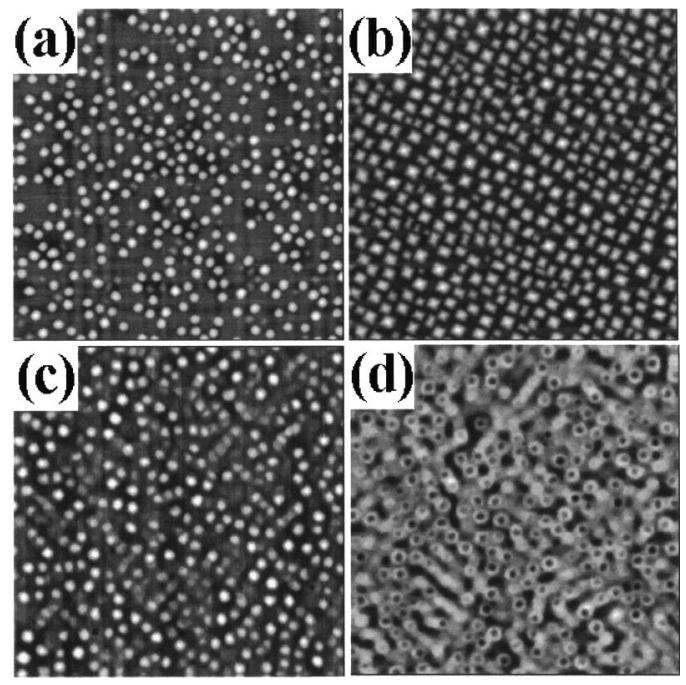

FIG. 1. AFM images $(2 \mu \mathrm{m} \times 2 \mu \mathrm{m})$ of Ge QDs capped with (a) 0, (b) 14, (c) 21 , and (d) 28 eq-MLs of Si.

1(d) shows the surface morphology after deposition of 28 eq-MLs Si. At this stage, the ring-like islands (nanorings) appeared to replace the larger QDs in the images shown. A three-dimensional image of nanorings is shown in Fig. 2. These nanorings have a height and diameter of $1.2 \pm 0.5$ and $65 \pm 6 \mathrm{~nm}$, respectively, with full width at half-maximum width measured to be $35 \pm 5 \mathrm{~nm}$. For deposition with 56 eqMLs $\mathrm{Si}$ capping layer, the ring-like structures tended to smooth out and were no longer evident in the AFM images. In addition, the rings were found to be of transient nature since they would disappear if the samples were kept in the growth chamber at $600^{\circ} \mathrm{C}$ for $10 \mathrm{~min}$.

A number of previous studies have demonstrated many interesting physical phenomena or optical properties of ringlike nanostructures in InAs/GaAs systems. ${ }^{12-14}$ In view of the transient nature of the nanorings in this work, the $\mathrm{Si} / \mathrm{Ge}$ ringlike islands captured by rapid cooling of the samples with appropriate amount of $\mathrm{Si}$ capping in this work shall provide a good framework to investigate the properties of unconventional $\mathrm{SiGe}$ quantum structures. ${ }^{15,16}$

Figures 3(a), 3(b), and 3(c) show XTEM images of the uncapped domes, truncated pyramids and nanorings, respectively. It is evident that Ge QDs reduced its heights with the
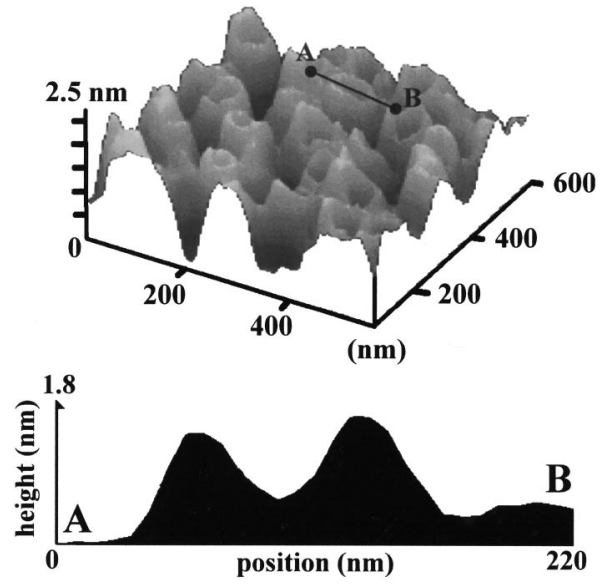

FIG. 2. Three-dimensional AFM image of the nanorings and the line profile

that it takes thicker SiGe than Si layer to induce the compa-
of a nanoring.
Downloaded $18 \mathrm{Dec} 2003$ to 140.112.5.250. Redistribution subject to AlP license or copyright, see http://ojps.aip.org/aplo/aplcr.js

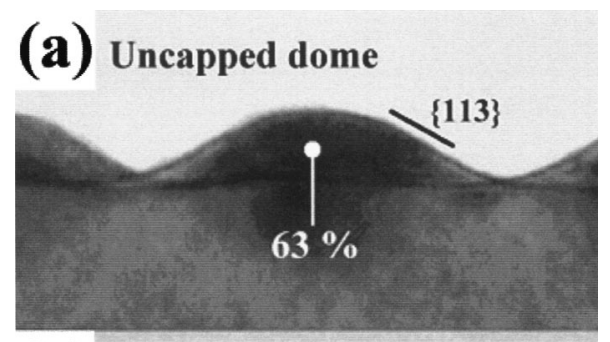

(b) Truncated pyramid
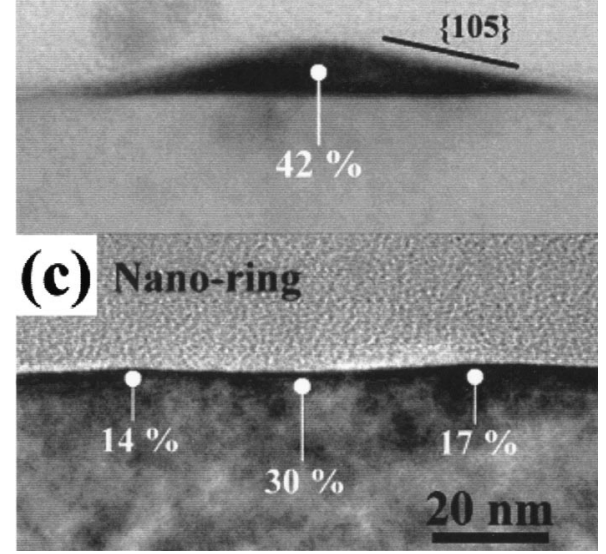

FIG. 3. XTEM images of the (a) Ge QDs without and (b) capped with 14 eq-MLs, and (c) 28 eq-MLs of Si: (a) and (c) are viewed along the [011] Si direction. The image in (b) was taken along [010] direction so that the profile of the pyramid base can be seen. The percentages marked in the figures refer to percentages of Ge determined by EDS.

increase of Si coverage. This evolution indicates that the intermixing between $\mathrm{Si}$ and Ge QDs tends to develop the stable (001) surface to replace the higher angle facets with higher energy as shown in Figs. 3(a) and 3(b). This result is consistent with those of the earlier studies for the Si overgrowth on the Ge QDs. ${ }^{9,10}$ With the increase of Si capping layer to 28 eq-MLs, the nanorings appear as shown in Fig. 3 (c). It is likely that due to the partial relaxation of Ge dot at the apex, a strain modulation was introduced on the surface layer. Above the Ge-rich dot, the lattice of Si-rich capping layer was laterally elongated and exhibits tensile strain. To reduce the accumulated strain energy, the $\mathrm{Si}$ atoms escaped from the severely strained regions to the relatively strain-free regions around the Ge-rich QDs and developed the nanorings on the surface. In the meantime, the intermixing between $\mathrm{Si}$ caping layer and Ge QDs continued. EDS measurement for the nanoring seen in Fig. 3(c) shows that the Ge compositions at the core and ridges are about $30 \%$ and $15 \%$, respectively. The results indicate that substantial intermixing of $\mathrm{Si}$ and Ge occurred during the formation of the nanorings. The intermixing between $\mathrm{Si}$ and $\mathrm{Ge}$ also leads to the relatively poor contrast of Ge QDs in both XTEM and planview TEM images.

In order to further elucidate the formation mechanism of the nanorings, a 40 eq- $\mathrm{ML} \mathrm{Si} \mathrm{Si}_{0.9} \mathrm{Ge}_{0.1}$ was grown on Ge QDs. As expected, the nanorings formed are of a smaller mean diameter of $42 \mathrm{~nm}$ owing to the reduction of mismatch strain between SiGe capping layer and Ge QDs. An example is shown in Fig. 4. We note that the selection of 40 eq-ML as the capping layer thickness was based on the understanding that it takes thicker $\mathrm{SiGe}$ than $\mathrm{Si}$ layer to induce the compa- 


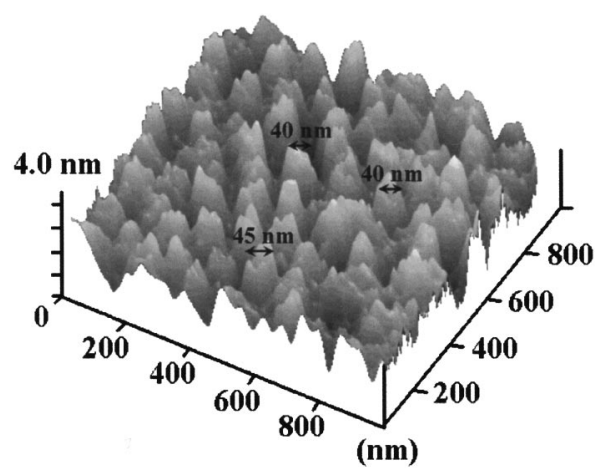

FIG. 4. AFM image of the nanorings in a SiGe-capped Ge QDs sample.

rable stress-induced effect. It is conceivable by varying the composition of the capping layer, the size of nanoring can be controlled.

From a comparison of Raman spectra of uncapped Ge QDs sample and blank Si wafer, the shift of $\mathrm{Ge}-\mathrm{Si}$ peak to lower wave numbers is generally caused by the presence of $\mathrm{Si}$ in the Ge QDs. In addition, the existence of high $\mathrm{Ge}-\mathrm{Si}$ peak intensity confirms the diffusion of Si into Ge QDs during growth. According to Kolobov et al.,${ }^{17}$ the Ge-rich area in the Ge QDs exists as a core of the dots encapsulated by the intermixed phase. It is well known that strain introduces the shifts of $\mathrm{Ge}-\mathrm{Ge}$ and $\mathrm{Ge}-\mathrm{Si}$ peak in the same direction toward higher wave numbers. ${ }^{18}$ The $\mathrm{Ge}-\mathrm{Si}$ peak of originally uncapped Ge QDs is shifted from 411.8 to $417.5 \mathrm{~cm}^{-1}$ when the Si capping layer increases to 21 eq-MLs as seen in Fig. 5. It can be attributed to increased strain as a result of the Si capping. With the increase of Si capping layer to 28 eq-MLs, the nanorings form simultaneously, the Ge-Si peak shifts to lower wave numbers by $\sim 1.1 \mathrm{~cm}^{-1}$ (from 417.5 to $416.4 \mathrm{~cm}^{-1}$ ). This peak shift implies that the accumulated strain stored in the $\mathrm{Si} / \mathrm{Ge}$ QDs system was partially relieved through the formation of nanorings on the surface and is consistent with the AFM results. It should be noted the intermixing between Si capping layer and Ge QDs would also shift the Ge-Si peak to lower wave numbers. Nevertheless, the intermixing between $\mathrm{Si}$ and Ge QDs has occurred at the

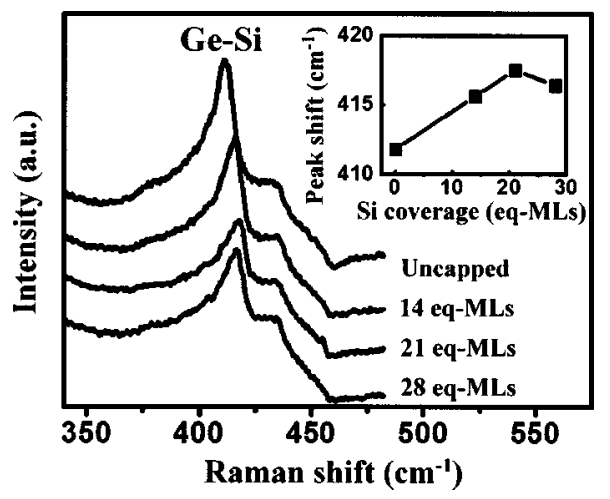

FIG. 5. Ge-Si peaks of the uncapped and Si-capped QDs near $410 \mathrm{~cm}^{-1}$ in the high dispersion Raman spectra. Inset shows the peak shift vs the number of capping layer. initial stage of $\mathrm{Si}$ encapsulation and continued during the $\mathrm{Si}$ capping process. This suggests that the shift to the lower wave number of $\mathrm{Ge}-\mathrm{Si}$ peak is more closely correlated with the formation of nanorings due to strain relief. Very recently, Cui et al. reported the observation of self-assembled SiGe quantum rings grown on $\mathrm{Si}(001)$ by molecular beam eptiaxy. ${ }^{19}$ Similar to the present study, they attributed the formation of SiGe quantum rings to the strain relief, SiGe alloying, and Ge segregation, while in our growth using the UHV/CVD system, Ge segregation was suppressed by the $\mathrm{H}$ passivation.

In summary, the evolution of the surface morphology and microstructures of Ge QDs on Si (001) during Si overgrowth have been investigated at different $\mathrm{Si}$ coverages. With increasing Si coverage to 28 eq-MLs, the uncapped Ge QDs were found to change their shapes dramatically from bimodal system to truncated pyramids, and eventually to the nanorings. Substantial intermixing between capping $\mathrm{Si}$ and Ge QDs is evident from both the EDS data and the contrast change in XTEM images. The Raman results demonstrate that the formation of nanorings is closely correlated with a strain-driven process. Moreover, it was found that the nanoring could be controlled by varying the composition of capping layers.

The research is supported by the Republic of China National Science Council Grant No. NSC 91-2215-E-007-015 and Ministry of Education Grant No. 91-E-FA04-1-4 as well as ERSO, ITRI.

${ }^{1}$ H. Sunamura, N. Usami, Y. Shiraki, and S. Fukatsu, Appl. Phys. Lett. 66, 3024 (1995).

${ }^{2}$ C. S. Peng, Q. Huang, W. Q. Cheng, J. M. Zhou, Y. H. Zhang, T. T. Sheng, and C. H. Tung, Phys. Rev. B 57, 8805 (1998).

${ }^{3}$ J. Wan, Y. H. Luo, Z. M. Jiang, G. Jin, J. L. Liu, K. L. Wang, X. Z. Liao, and J. Zou, Appl. Phys. Lett. 79, 1980 (2001).

${ }^{4}$ B.-C. Hsu, S. T. Chang, C.-R. Shie, C.-C. Lai, P. S. Chen, and C. W. Liu, IEDM 2002, 91 (2002).

${ }^{5}$ Z. Pei, C. S. Liang, L. S. Lai, Y. T. Tseng, Y. M. Hsu, P. S. Chen, S. C. Lu, C. M. Liu, M.-J. Tsai, and C. W. Liu, IEDM 2002, 271 (2002).

${ }^{6}$ F. M. Ross, J. Tersoff, and R. M. Tromp, Phys. Rev. Lett. 80, 984 (1998).

${ }^{7}$ G. Medeiros-Ribeiro, A. M. Brathovski, T. I. Kamins, D. A. A. Ohlberg, and R. S. Williams, Science 279, 353 (1998).

${ }^{8}$ T. Brunhes, P. Boucaud, S. Sauvage, F. Aniel, J.-M. Lourtioz, C. Hernandez, Y. Campidelli, O. Kermarrec, D. Bensahel, G. Faini, and I. Sagnes, Appl. Phys. Lett. 77, 1822 (2000).

${ }^{9}$ P. Sutter and M. G. Lagally, Phys. Rev. Lett. 81, 3471 (1998).

${ }^{10}$ A. Rastelli, M. Kummer, and H. von Kanel, Phys. Rev. Lett. 87, 256101 (2001).

${ }^{11}$ T. I. Kaimins, G. Mederiros-Ribeiros, D. A. A. Ohlberg, and R. S. Williams, J. Appl. Phys. 85, 1159 (1999).

${ }^{12}$ D. Granados and J. M. Garía, Appl. Phys. Lett. 82, 2401 (2003).

${ }^{13}$ A. Lorke, R. J. Luyken, A. O. Govorov, J. P. Kotthaus, J. M. Garcia, and P. M. Petroff, Phys. Rev. Lett. 84, 2223 (2000).

${ }^{14}$ J. C. Lin and G. Y. Guo, Phys. Rev. B 65, 035304 (2001).

${ }^{15}$ J. Liu, A. Zaslavsky, B. R. Perkins, C. Aydin, and L. B. Freund, Phys. Rev. B 66, 161304 (2002).

${ }^{16}$ J. Liu, A. Zaslavsky, and L. B. Freund, Phys. Rev. Lett. 89, 096804 (2002).

${ }^{17}$ A. V. Kolobov, K. Morita, K. M. Itoh, and E. E. Haller, Appl. Phys. Lett. 81, 3855 (2002).

${ }^{18}$ F. Cerdeira, A. Pinczuk, J. C. Bean, B. Batlogg, and B. A. Wilson, Appl. Phys. Lett. 45, 1138 (1984).

${ }^{19}$ J. Cui, Q. He, X. M. Jiang, Y. L. Fang, X. J. Yang, F. Xue, and Z. M. Jiang, Appl. Phys. Lett. 83, 2907 (2003). 